\title{
On the exchange property for the Laplace transform
}

\author{
Abhishek Singh, Deshna Loonker and P.K. Banerji \\ Department of Mathematics, Faculty of Science, J.N.V. University, Jodhpur - 342 005, India \\ banerjipk@yahoo.com
} \begin{abstract}
2000 Mathematics Subject Classifications: 46F10, 46F99, 44A10.
Introduction

The concept of Boehmians is motivated by the regular operator introduced by Boehme (Boehme, 1973), which form a subalgebra of the field of Mikusiński operators and thus they include only such functions whose support is bounded from the left. The theory of Boehmians (quotient of sequences), its properties and different classes of Boehmian spaces can be studied in (Mikusiński, 1995; Atanasiu \& Mikusiński, 2005; Banerji \& Loonker, 2006).Tempered Boehmians is a natural extension of tempered distribution which, therefore, makes it possible to define an extension of the Fourier transform for this class of Boehmians. The Fourier transform of a tempered Boehmian is a distribution. A
\end{abstract}

Abstract

In this paper we investigate the exchange property for the Laplace transform by using the relation between the Fourier transform and the Laplace transform. Simplified construction of tempered Boehmians is also presented.

Keywords: Distribution, Boehmians, tempered distribution, tempered Boehmians, Laplace transform.

continuous function $f: R^{n} \rightarrow C$ is called slowly increasing if there is a polynomial $p$ on $R^{N}$ such that $|f(x)| \leq p(x)$ for all $x \in R^{N}$. The space of slowly increasing function will be denoted by $J\left(R^{N}\right)$ or simply by

$J$. An infinitely differentiable function $f: R^{n} \rightarrow C$ is called rapidly decreasing if

$$
\sup _{\|\alpha\| \leq m} \sup _{x \in R^{N}}\left(1+x_{1}^{2}+\ldots+x_{N}^{2}\right)^{m}\left|D^{\alpha} f(x)\right|<\infty,
$$

for every nonnegative integer $m$, where $x=\left(x_{1}, x_{2} \ldots x_{N}\right)$, $\alpha=\left(\alpha_{1}, \ldots \alpha_{N}\right), \alpha_{N}$ 's are non negative integer,

$|\alpha|=\alpha_{1}+\ldots+\alpha_{N}$, and

$D^{\alpha}=\frac{\partial^{|\alpha|}}{\partial x^{\alpha}}=\frac{\partial^{|\alpha|}}{\partial x_{1}^{\alpha_{1}} \ldots \partial x_{N}^{\alpha_{N}}}$.

The space of rapidly decreasing functions is denoted by $S\left(R^{V}\right)$ or simply by $S$. If $f \in J$ and $\varphi \in S$, then the convolution

$$
(f * \varphi)(x)=\int_{R^{N}} f(u) \varphi(x-u) d u
$$

is well defined and $f * \varphi \in J$. A sequence $\varphi_{n} \in S$ is called a delta sequence if it satisfies the following conditions:

Research article.

CIndian Society for Education and Environment (iSee) (i) $\int_{R^{N}} \varphi_{n}(x) d x=1$, for all $n \in N$

(ii) $\int_{R^{N}}\left|\varphi_{n}(x)\right| d x \leq M$, for some constant $M$ and for all $n \in N$

(iii) $\lim _{n \rightarrow \infty} \int_{\|x\| \geq \varepsilon}\|x\|^{k}\left|\varphi_{n}(x)\right| d x=0, \quad$ for every $k \in N$ and $\varepsilon>0$.

We consider $f_{n} \in J,\left\{\varphi_{n}\right\}$ is a delta sequence under usual notations. Then the space of equivalence classes of quotients of sequences will be denoted by $\beta_{J}$, elements of which are called tempered Boehmian. If $F=\left[f_{n} / \varphi_{n}\right] \in \beta_{J}$ and $f_{n} \in S, \forall n \in N$, then $F$ is called rapidly decreasing Boehmian, denoted by $\beta_{S}$.

In the following section we employ the relation between the Fourier transform and the Laplace transform (Banerji \& Loonker, 2006) and investigate the exchange property for the Laplace transform. In subsequent section algebraic properties and convergence is proved for this relation on the tempered Boehmians.

In what follows, we will denote by $S^{\prime}$ the space of tempered distributions, that is, the space of continuous linear functionals on $S$, If $f \in S^{\prime}$ and $\varphi \in S$, then the convolution $f * \varphi$ is defined as $(f * \varphi)(x)=f\left(\varphi_{x}\right)$, where $\varphi_{x}(z)=\varphi(x-z)$. It can be shown that, if $\mathrm{f} \in g^{\prime}$ and $\varphi \in g$, then $f^{*} \varphi \in J$. The Laplace transform of a tempered distribution $f$, denoted by $L f$, is the functional on $\rho$ defined by $L f(\varphi)=f(L \varphi)$, where $L \varphi$ is the Laplace transform of $\varphi$.

We will denote by $A$ the collection of all families of pairs $\left\{\left(f_{i}, \varphi_{i}\right)\right\}_{l}$, where / is an index set, $f_{i} \in S^{\prime}$ and $\varphi_{i} \in S$ for all $i \in l$, satisfying the exchange property such that $P\left(\left\{\varphi_{i}\right\}_{I}\right)=\varnothing$. If $\left(\varphi_{i}\right)$ is a delta sequence, then $P\left(\left\{\varphi_{i}\right\}_{N}\right)=\varnothing$. 
The Laplace transform and the exchange property

The classical definiton of the Laplace transform (Banerji \& Loonker, 2006) of a function $f$ is

$L[f](p)=\int_{R_{+}^{n}} e^{-p t} f(t) d t, \quad p \in C$.

The relation between the Laplace and the Fourier transform is given by (Banerji \& Loonker, 2006)

$L[f](p)=\mathrm{F}\left[e^{-\sigma t} f(t)\right](-\tau)$,

where $f \in E_{\Gamma}, p \in H_{\Gamma}, e^{\sigma t} f(t) \in L_{1}\left(R^{N}\right)$ and $\sigma \in R^{n}$, $\Gamma \geq a$. Further, $E_{\Gamma}\left(\Gamma \in R^{N}\right)$ is the set of function from $R^{n}$ into $C^{n}$ (which are $n$-dimensional Euclidean and complex planes, respectively) whereas,

$H_{\Gamma}=\left\{p: p \in C^{n}, \quad \operatorname{Re}(p)>\Gamma\right\}$

and $R_{+}^{n}=\left\{x: x \in R^{n}, x>0\right\}$.

The inversion formula for the relation (6) is given by

$f(t)=2 \pi \int_{\Gamma} e^{p t} L[f](p) d p, \quad \Gamma \in R_{+}^{n}, \quad \Gamma \geq a$.

For a family $\left\{\varphi_{i}\right\}_{i \in I}=\left\{\varphi_{i}\right\}_{I}, \varphi_{i} \in S$ for all $i \in l$, we define

$$
P\left(\left\{\varphi_{i}\right\}_{I}\right)=\left\{x \in R^{N}: L\left[\varphi_{i}\right](x)=0, \quad \forall i \in I\right\}
$$

A family of pairs $\left\{\left(f_{i}, \varphi_{i}\right)\right\}$, where $f_{i} \in S^{\prime}$ and $\varphi_{i} \in S$, is said to have the exchange property if

$f_{i} * \varphi_{k}=f_{k} * \varphi_{i}, \quad \forall i, k \in I$.

Definition 1. If $\left\{\left(f_{i}, \varphi_{i}\right)\right\}_{,} \in A$, then the unique $F \in D^{\prime}\left(R^{N}\right)$ such that $L f_{i}=L \varphi_{i} F$ for all $\quad i \in I$ will be denoted by $L\left\{\left(f_{i}, \varphi_{i}\right)\right\}_{1}$.

Let $\left\{\left(f_{i}, \varphi_{i}\right)\right\}_{I},\left\{\left(g_{k}, \psi_{k}\right)\right\}_{k} \in A$.

If $f_{i} * \psi_{k}=g_{k} * \varphi_{i}$ for all $i \in /$ and $k \in K$, then we write $\left\{\left(f_{i}, \varphi_{i}\right)\right\}_{I} \sim\left\{\left(g_{k}, \psi_{k}\right)\right\}_{K}$. This relation is clearly symmetric and reflexive. We will show that it is also transitive.

$$
\begin{aligned}
& \text { Let }\left\{\left(f_{i}, \varphi_{i}\right)\right\}_{I},\left\{\left(g_{k}, \psi_{k}\right)\right\}_{K},\left\{\left(h_{l}, \gamma_{l}\right)\right\}_{L} \in A \text {. } \\
& \text { If } \quad\left\{\left(f_{i}, \varphi_{i}\right)\right\}_{I} \sim\left\{\left(g_{k}, \psi_{k}\right)\right\}_{K} \\
& \left\{\left(g_{k} * \psi_{k}\right)\right\}_{K} \sim\left\{\left(h_{l} * \gamma_{l}\right)\right\}_{L} \text {, then } \\
& f_{i} * \psi_{k}=g_{k} * \varphi_{i}, \quad g_{k} * \gamma_{l}=h_{l} * \psi_{k}
\end{aligned}
$$

for all $i \in l, k \in K, l \in L$. Therefore,

$$
f_{i} * \psi_{k} * \gamma_{l}=g_{k} * \varphi_{i} * \gamma_{l}, g_{k} * \gamma_{l} * \varphi_{i}=h_{i} * \psi_{k} * \varphi_{i}
$$

for all $i \in l, k \in K, l \in L$. Since $*$ is commutative, we have $f_{i} * \gamma_{l} * \psi_{k}=h_{i} * \varphi_{i} * \psi_{k}$.

Now fix $i \in /$ and $I \in L$. Since $P\left(\left\{\psi_{k}\right\}_{K}\right)=\varnothing$ and (13) holds for every $k \in K$, we conclude that $f_{i} * \gamma_{l}=h_{l} * \varphi_{i}$ for all $i \in /$ and $/ \in L$, which means that $\left\{\left(f_{i}, \varphi_{i}\right)\right\}_{I} \sim\left\{\left(h_{l}, \gamma_{l}\right)\right\}_{L}$.

Theorem 1: If a family of pair $\left\{\left(f_{i}, \varphi_{i}\right)\right\}_{I}$ has the exchange property and $\Omega=P\left\{\left(\varphi_{i}\right\}_{I}\right)^{c}$ (the complement of $P\left\{\left(\varphi_{i}\right\}_{I}\right)$ in $\left.R^{\prime \prime}\right)$, then there exists a unique $F \in D^{\prime}(\Omega)$ such that

$L\left[f_{i}\right]=F L\left[\varphi_{l}\right], \quad \forall i \in I$.

Proof : For every $x \in \Omega$ there exists $i \in /$ and $\varepsilon>0$ such that $\left|L \varphi_{i}(x)\right|>\varepsilon$ in an open neighbourhood of $x$. Then we can define $F=L f_{i} / L \varphi_{i}$ in that neighbourhood. Let for some $\varepsilon>0$, we have $\left|L \varphi_{i}(x)\right|>\varepsilon$ for all $x \in U$ and $\left|L \varphi_{k}(x)\right|>\varepsilon$ for all $x \in V$, where $U$ and $V$ are open sets. Since $f_{i} * \varphi_{k}=f_{k} * \varphi_{i}$, we have

$$
L f_{i} L \varphi_{k}=L f_{k} L \varphi_{i} \text { and } \frac{L f_{i}}{L \varphi_{i}}=\frac{L f_{k}}{L \varphi_{k}},
$$

on $U \cap V$. This shows that $F$ is a unique function.

Theorem 2: $\left\{\left(f_{i}, \varphi_{i}\right)\right\}_{I} \in A$ if and only if there exists a unique $F \in D^{\prime}\left(R^{N}\right)$ such that $L f_{i}=L \varphi_{i} F$ for all $i \in l$.

Proof : For any $i, k \in I$, we have $L f_{i} L \varphi_{k}=F L \varphi_{i} L \varphi_{k}$, [ by Theorem 1]

$=F L \varphi_{k} L \varphi_{i} \quad=L f_{k} L \varphi_{i}$.

This proves that the existence of such a function $F \in D^{\prime}$ $\left(R^{N}\right)$ implies and justifies the exchange property. This completes the proof of the theorem.

Theorem 3: There exists $\left\{\left(f_{i}, \varphi_{i}\right)\right\}_{I} \in A$, for every $F \in D^{\prime}$ $\left(R^{N}\right)$ and such that $F=L\left(\left\{\left(f_{i} \varphi_{i}\right)\right\}_{I}\right)$.

Proof: Since $D\left(R^{V}\right)$ denotes the space of smooth function with compact support, there exists a total sequence $\left\{\varphi_{i}\right\}_{N}$ such that $L \varphi_{i} \in D\left(R^{N}\right)$ for all $/ \in N$. Then for every / $\in N$, there is $f_{i} \in S$ such that $L f_{i}=L \varphi_{i} F$. Clearly $\left\{\left(f_{i}, \varphi_{i}\right)\right\}_{N} \in A$ and $F=L\left(\left\{\left(f_{i}, \varphi_{i}\right)\right\}_{N}\right)$. This completes the proof of theorem.

Definition 2 (Atanasiu \& Mikusiński, 2005): Let $\left\{U_{i j}\right.$, be an open covering of $R^{N}$ and let $\left\{\varphi_{i}\right\}_{I}$ be such that $\left|L \varphi_{i}(x)\right|>0$ for $x \in U_{i}$. A family $\left\{\varphi_{i}\right\}_{I}$ such that $P\left(\left\{\varphi_{i}\right\}_{I}\right)=\varnothing$ will be called total.

Lemma 1 (Atanasiu \& Mikusiński,, 2005):

If $\left\{\varphi_{i}\right\}_{I}$ and $\left\{\psi_{k}\right\}_{K}$ are total, then $\left\{\varphi_{i}^{*} \psi_{k}\right\}_{I \times K}$ is total.

Theorem 4: Let $\left\{\left(f_{i}, \varphi_{i}\right)\right\}_{I},\left\{\left(g_{k}, \psi_{k}\right)\right\}_{K} \in A$. Then $\left\{\left(f_{i}, \varphi_{i}\right)\right\}_{I} \sim\left\{\left(g_{k}, \psi_{k}\right)\right\}_{K}$ if and only
Singh et al. Indian J.Sci.Technol. 
if $L\left(\left\{\left(f_{i}, \varphi_{i}\right)\right\}_{I}\right)=L\left(\left\{\left(g_{k}, \psi_{k}\right)\right\}_{K}\right)$.

Proof : $F=L\left(\left\{\left(f_{i}, \varphi_{i}\right)_{I}\right\}\right)$ and $G=L\left(\left\{\left(g_{k}, \psi_{k}\right)\right\}_{K}\right)$.

If $\left.\left\{\left(f_{i}, \varphi_{i}\right)\right\}_{I} \sim\left\{g_{k}, \psi_{k}\right)\right\}_{K}$,

then $F L \varphi_{i} L \psi_{k}=L f_{i} L \psi_{k}=L g_{k} L \varphi_{i}$

$F L \varphi_{i} L \psi_{k}=G L \psi_{k} L \varphi_{i}, \quad \forall i \in I, k \in K$.

Hence $F=G$, by Lemma 1. Now assume $F=G$. Then

$L f_{i} L \psi_{k}=F L \varphi_{i} L \psi_{k}=G L \psi_{k} L \varphi_{i}=L g_{k} L \varphi_{i}, \quad \forall i \in I, k \in K$. (18)

Hence $\left\{\left(f_{i}, \varphi_{i}\right)\right\}_{I} \sim\left\{\left(g_{k}, \psi_{k}\right)\right\}_{K}$. This completes the proof of the theorem.

Theorem 5: There exists a delta sequence $\left(\varphi_{n}\right)$ such that for every $T \in \beta, T=\left[\left\{\left(f_{n}, \varphi_{n}\right)\right\}_{N}\right]$ for some $f_{n} \in J$.

Proof: Let $\left(\psi_{n}\right)$ be a delta sequence such that $L \psi_{n} \in D$. Then for any $T \in \beta$, we have $L T L \psi_{n} \in S^{\prime}$, since $L T \in D^{\prime}$. Consequently, $L T L \psi_{n}=L g_{n}$ for some $g_{n} \in S^{\prime}$. It is easy to check that $T=\left[\left\{\left(g_{n} * \psi_{n}, \psi_{n} * \psi_{n}\right)\right\}_{N}\right]$. Since $f_{n}=g_{n} * \psi_{n} \in J$ and $\left(\varphi_{n}\right)=\left(\psi_{n} * \psi_{n}\right)$ is a delta sequence, where $\left(\varphi_{n}\right)$ does not depend on $T$, hence the theorem is proved.

Algebraic properties and convergence

$\beta_{J}$ becomes a vector space with the addition operation, defined by

$\left[\left\{\left(f_{i}, \varphi_{i}\right)\right\}_{I}\right]+\left[\left\{\left(g_{k}, \psi_{k}\right)\right\}_{K}\right]=\left[\left\{\left(f_{i} * \psi_{k}+g_{k} * \varphi_{i}, \varphi_{i} * \psi_{k}\right)\right\}_{I \times K}\right](19)$

Moreover, multiplication by a scalar and the operation $*$ are defined by

$\lambda\left[\left\{\left(f_{i}, \varphi_{i}\right)\right\}_{I}\right]=\left[\left\{\left(\lambda f_{i}, \varphi_{i}\right)\right\}_{I}\right], \quad \lambda \in C$.

If $\left[\left\{\left(f_{i}, \varphi_{i}\right)\right\}_{I}\right], \quad\left[\left\{\left(g_{k}, \psi_{k}\right)\right\}_{K}\right] \in \beta_{J}$ and $g_{k} \in S$ for all $k$ $\in K$, then for the operation $*$ we can define

$\left[\left\{\left(f_{i}, \varphi_{i}\right)\right\}_{I}\right] *\left[\left\{\left(g_{k}, \psi_{k}\right)\right\}_{k}\right]=\left[\left\{\left(f_{i} * g_{k}, \varphi_{i} * \psi_{k}\right)\right\}_{I \times K}\right]$

Definition 3 (Atanasiu \& Mikusiński, 2005):

Let $T_{0}, T_{1}, T_{2}, \ldots \in \beta_{\jmath}$. Then the sequence $\left(T_{n}\right)$ is said to converge to $T_{0}$, which is written as $T_{\mathrm{n}} \rightarrow T_{0}$ if there exists a total family $\left\{\varphi_{i}\right\}_{\text {, such that }}$

(a)there exists tempered distribution $f_{i, n}$

where $i \in /$ and $n \in N$ such that

$T_{n}=\left[\left\{f_{i, n}, \varphi_{i}\right\}_{I}\right]$ for all $n=0,1,2, \ldots$,

(b) $f_{i, n} \rightarrow f_{i, 0}$ in $S^{\prime}$ as $n \rightarrow \infty$ for every $i \in L$.

Theorem 6: The Laplace transform is an isomorphism from $\beta_{J}$ to $D^{\prime}$.

Proof: Since $T_{\mathrm{n}} \rightarrow T_{0}$ in $\beta_{J}$ if and only if $T_{m}-T_{0} \rightarrow 0$, it suffices to prove the continuity at 0 . Let $T_{\mathrm{n}} \rightarrow 0$ is in $\beta_{J}$. Then there exists tempered distribution $f_{i, n}$ where $i \in /$ and $n \in N$ such that $T_{n}=\left[\left\{f_{i, n}, \varphi_{i}\right\}_{I}\right]$ for all $n=1,2, \ldots$ and $f_{i, n} \rightarrow 0$ in $S^{\prime}$ as $n \rightarrow \infty$ for every $i \in I$. If $\psi \in D$, then there are $i_{1}, \ldots, i_{k}$ such that

Research article.

(C) Indian Society for Education and Environment (iSee)
.Then

$$
\begin{aligned}
& \lim _{n \rightarrow \infty} L T_{n} \psi=\lim _{n \rightarrow \infty} \sum_{m=1}^{k}\left(L T_{n} L \varphi_{i_{m}}\right) \frac{\overline{L \varphi_{i_{m}}} \psi}{\sum_{m=1}^{k}\left|L \varphi_{i_{m}}\right|^{2}} \\
& =\sum_{m=1}^{k}\left(\lim _{n \rightarrow \infty} L f_{i_{m}, n}\right) \frac{\overline{L \varphi_{i_{m}}} \psi}{\sum_{m=1}^{k}\left|L \varphi_{i_{m}}\right|^{2}}=0
\end{aligned}
$$

because $\lim _{n \rightarrow \infty} L f_{i, n}=0$ for $\forall i \in I$, due to the continuity of the Laplace transform in $S^{\prime}$. This proves the continuity of $L$ : $\beta_{J} \rightarrow D^{\prime}$, because $\lim _{n \rightarrow \infty} L T_{n} \psi=0$ in $\mathcal{S}^{\prime}$ for every $\psi \in D$, implies $\lim _{n \rightarrow \infty} L T_{n}=0$ in $D^{\prime}$.

Now, assume $\lim _{n \rightarrow \infty} L T_{n}=0$ in $D^{\prime}$. By Theorem 5, there exists a delta sequence $\left(\varphi_{i}\right), i \in N$ such that for every $n$ $\in N$, we have $T_{n}=\left[\left\{\left(f_{i, n}, \varphi_{i}\right)\right\}_{N}\right]$ for some $f_{i, n} \in J$. Let $\left(\psi_{k}\right), k \in N$ be a delta sequence such that $L \psi_{k} \in D$ for every $k \in N$. Then $\lim _{n \rightarrow \infty} L T_{n} L \varphi_{i} L \psi_{k}=0$. in $\mathcal{S}^{\prime}$ for every $i$, $k \in N$.

Since $\quad L T_{n} L \varphi_{i}=f_{i, n} \quad \forall i, k \in N$,

$\lim _{n \rightarrow \infty} L f_{i, n} L \psi_{k}=0$ in $S^{\prime}$

which implies $\lim _{n \rightarrow \infty} f_{i, n} * \psi_{k}=0$, in $S^{\prime}$ But

$T_{n}=\left[\left\{\left(f_{i, n}, \varphi_{i}\right)\right\}_{I}\right]=\left[\left\{\left(f_{i, n} * \psi_{k}, \varphi_{k} * \psi_{k}\right)\right\}_{I \times K}\right]$

for all $n=0,1,2, \ldots$. Thus we have $T_{n} \rightarrow 0$ in $\beta_{J}$. This proves the theorem.

Acknowledgement

This work is partially supported by the JNV University Research Scholarship No. 899, sanctioned to the first author (AS), the DST (SERC) Fast Track for Young Scientist, Sanction No. SR/FTP/MS-22/2007, to the second author (DL) and the financial assistance under Emeritus Fellow honorarium of UGC, Sanction No. F.6-6/2003/(SA-II), to the third author (PKB).

\section{References}

1. Atanasiu D and Mikusiński $P$ (2005) On the Fourier transform and the exchange property. Int/. J. Math. Math. Sci. 16, 2579-2584.

2. Banerji PK and Loonker D (2006) Laplace transform for Integrable Boehmians. Bull. Cal. Math. Soc. 98 (5), 465-470.

3. Boehme TK (1973) The support of Mikusinski operators. Trans. Amer. Math. Soc. 176, 319-334.

4. Mikusiński P (1995) Tempered Boehmians and ultradistributions. Proc. Amer. Math. Soc. 123, 813-817.

5. Zemanian AH (1987) Distribution theory and transform analysis, Dover Publ., Inc., NY. 